\title{
Evaluation of the Single-Dose Toxicity of TA Pharmacopuncture in Rats
}

\author{
Ji Hye Hwang, ${ }^{1 *}$ Hyo Won Jung, ${ }^{2}$ Chul Jung ${ }^{3 *}$ \\ ${ }^{1}$ Department of Acupuncture \& Moxibustion Medicine, College of Korean Medicine, Gachon University, Seongnam 13120, Republic of Korea \\ ${ }^{2}$ College of Korean Medicine, Dongguk University, Gyeongju 38066, Republic of Korea \\ ${ }^{3}$ Namsangcheon Korean Medicine Clinic, Seoul 06656, Republic of Korea
}

\section{Key Words}

safety, single-dose intramuscular toxicity, TA pharmacopuncture, traditional medicine, acupuncture

\section{Abbreviations}

TA: a seven-herb extract consisting of Scutellariae Radix, Phellodendri Cortex, Pulsatillae Radix, Sophorae Tonkinensis Radix et Rhizoma, Aucklandiae Radix, Aquilariae Lignum, and Carthami Fructus

\begin{abstract}
Objectives: TA is a polyherbal extract comprising seven herbs, typically used for the pharmacopuncture treatment of patients with traffic accident- related injuries and musculoskeletal diseases. This animal study was conducted to evaluate the safety of the TA extract, using a single-dose toxicity test.
\end{abstract}

Methods: The dose range and sampling time were first established. Six- week-old Sprague-Dawley rats were administered $1.0 \mathrm{~mL}$ of TA or normal saline (control), intramuscularly, for the single-dose toxicity test. The general condition, mortality, and histology of all rats were observed for 2 weeks.

Results: No abnormal symptoms or deaths were observed in any group. The body weights of the rats in the TA and control groups were similar. No significant differenc-

Received: Feb 11, 2019 Reviewed: May 30, 2019 Accepted: Sep 03, 2019 es in histopathology were observed between the groups. Conclusion : Our study indicates that $1.0 \mathrm{~mL}$ of TA extract may be safely administered for pharmacopuncture for treatment of patients in traditional medicine clinics.

\section{Introduction}

The safety and efficacy of medicinal plants and their formulae used in Korean medicine (KM) have been proven by long-term clinical experience. However, there is a misconception that these medications are hepatotoxic or nephrotoxic, which may lead to patients avoiding traditional medicine for treatment. Thus, despite the clinical and empirical proof of safety, there remains a need to demonstrate the safety of this treatment through scientific toxicity evaluation. Pharmacopuncture, an acupoint injection used in traditional Chinese medicine and TKM, is a new method of acupuncture involving the injection of herbal medicine. Pharmacopuncture is considered to be faster and more effective than oral administration, because it can deliver the pharmacological agent to the target tissue/ organ directly, without passing through the digestive system $[1,2]$; therefore, it is often used to provide quick analgesia. TA is a polyherbal extract used for pharmacopuncture. It consists of Scutellariae Radix, Phellodendri Cortex, Pulsatillae Radix, Sophorae Tonkinensis Radix et Rhizoma, Aucklandiae Radix, Aquilariae Lignum, and Carthami Fructus and was thus, named for its principal use in treating traffic accident-relat- (c) This is an Open-Access article distributed under the terms of the Creative Commons Attribution Non-Commercial License (http://creativecommons.org/licenses/by-nc/4.0/) which permits unrestricted noncommercial use, distribution, and reproduction in any medium, provided the original work is properly cited.

@ This paper meets the requirements of KS X ISO 9706, ISO 9706-1994 and ANSI/NISO Z39.48-1992 (Permanence of Paper).
Corresponding Author

Chul Jung. Namsangcheon Korean Medicine Clinic, Seoul 06656, Republic of Korea. E-mail: jcnu2000@daum.net

Ji Hye Hwang. Department of Acupuncture \& Moxibustion Medicine, College of Korean Medicine, Gachon University, Seongnam 13120, Republic of Korea.

E-mail: jhbori@nate.com

(c) 2019 Korean Pharmacopuncture Institute

http://www.journal.ac 
ed injuries. TA consists of raw plant-based materials only, with an assured stable supply, since Bovis Calculus-Ursi Fel- Moschus extracts [BUM or V], the animal-based extracts which are used to treat acute inflammatory and painful diseases in TKM, are difficult to procure, owing to the international trade conventions regarding endangered species of wild fauna and flora. [3,4]

In vitro studies have reported that TA inhibits the production of nitric oxide and inflammatory cytokines, such as IL-6 and TNF- $\alpha$. [3] The TA extract was developed in 2015 to treat musculoskeletal diseases and traffic-accident injuries in clinical practice. Thus, it has not been widely used to date, and few clinical studies of its therapeutic effects on thoracic pain induced by seat-belts in traffic accidents [5] and frequent sprains [6] have been reported. Although the safety of the TA extract has been confirmed by clinical experience, its efficacy and safety require further scientific verification, before it can be used widely in clinical practice.

Our earlier studies confirmed the safety of TA by a bacterial reverse mutation test and by assessing in vitro chromosomal abnormalities in TA- treated mammalian-cultured cells $[7,8]$. However, since various types of toxicological data are required to recognize the safety of herbal medicines, additional experimental studies on the safety of TA are required. Therefore, in this study, we investigated the safety of TA using a single-dose intramuscular toxicity test in a murine model.

\section{Material and methods}

\subsection{Preparation of TA extract and control materials}

TA consists of seven herbs (Table 1). All materials were purchased from an herbal materials company (Jayeondameun, Yangju, Korea). The TA extract was manufactured as the finished product $(60.5 \mathrm{mg} / \mathrm{mL}$ in a sealed vial) using water and alcohol $(\mathrm{v} / \mathrm{v}=1: 1)$ at an extramural facility, meeting Korean Good Manufacturing Practice standards (Namsangcheon Herbal Medicine Dispensary, Yongin, Korea). The TA extract was stored in a refrigerator at $4.2-5.4^{\circ} \mathrm{C}$. Normal saline was used as a control, in the single-dose intramuscular toxicity test.

Table 1 Composition of the TA extract

\begin{tabular}{lcc}
\hline \multicolumn{1}{c}{ Herbal name } & Scientific name & $\begin{array}{r}\text { Ratio } \\
(\mathrm{mg} / \mathrm{ml})\end{array}$ \\
\hline Scutellariae Radix & Scutellaria baicalensis & 10 \\
Phellodendri Cortex & Phellodendron amurense & 10 \\
Pulsatilla Koreana & Pulsatilla koreana & 10 \\
$\begin{array}{l}\text { Sophorae Subprostratae } \\
\text { Radix }\end{array}$ & Sophora tonkinensis & 10 \\
Aucklandiae Radix & Aucklandia lappa & 5 \\
Aquilaria agallocha & Aquilaria agallocha & 0.5 \\
Carthami Tinctorii Fructus & Carthamus tinctorius L & 15 \\
\hline
\end{tabular}

\subsection{Experimental animals}

For the single-dose intramuscular toxicity test, SpragueDawley (SD) rats (6-weeks-old, male, and female, $n=5$, per group) were purchased from Orient Bio Inc (Seongnam, Korea). The body weight ranged from 190.1-206.2 g for the male and 147.4-163.4 g for the female rats, respectively. When the animals first arrived, a visual inspection was performed and their weight was recorded with electronic scales (CP3202S, Sartorius, Gottingen, Germany).

The general condition and changes in the animals were checked, once daily, during 1 week of acclimatization, and all the animals were judged to be normal. SD rats were housed under controlled environmental conditions with an ambient temperature of $22.5 \pm 2^{\circ} \mathrm{C}$, relative humidity of $54 \pm 6 \%, 12$-h/12-h light/dark cycle, and free access to food (Teklad Certified Irradiated Global 18\% Protein Rodent Diet 2918C, Envigo, Huntingdon, UK) and water. The mice were divided into two groups after the acclimatization period.

The animal were cared for and treated according to the Korean National Institute of Health and the Korean Academy of Medical Sciences guidelines. This study was conducted at Biotoxtech (Cheongwon, Korea) under the Good Laboratory Practice regulations [9], with approval of the Institutional Animal Ethics Committee of Biotoxtech (No. 170574, 170549).

\subsection{Single-dose intramuscular toxicity test in Sprague-Dawley rats}

\subsubsection{Administration and group designation}

Since the muscles are the target tissues for pharmacopuncture, the rats were administered TA extract was via intramuscular injection. The expected clinical dose of the test extract, $\mathrm{TA}$, is 0.1 to $1.0 \mathrm{~mL}(0.1 \mathrm{~mL} /$ time $)$ in humans. In a preliminary test (Biotoxtech Study No.: B17513P1), no mortalities were observed after intramuscular injection with a single dose of $0.25,0.5$, and $1.0 \mathrm{~mL}$ of TA in male and female rats. Based on the preliminary test, the dose of TA was set to $1.0 \mathrm{~mL}$ in this study (G2) and the same amount of saline was administered in the control group (G1) (Table 2). Ten male and 10 female rats, with body weights closest to the mean weight were selected for this experiment. The selected animals were divided into two groups (5 rats of each sex per group) and were administered intramuscular injections into the muscles of the left and the right femur, with a total dose of $1.0 \mathrm{~mL}$ per animal.

Table 2 Group designation of a single-dose intramuscular toxicity test in rats

\begin{tabular}{|c|c|c|c|c|c|}
\hline \multirow{2}{*}{\multicolumn{2}{|c|}{ Group }} & \multirow{2}{*}{$\begin{array}{c}\text { Dose } \\
\text { (mL/animal) }\end{array}$} & \multirow{2}{*}{$\begin{array}{l}\text { Dose amount } \\
\text { (mL/animal) }\end{array}$} & \multicolumn{2}{|c|}{ Number of animals (Subject number) } \\
\hline & & & & Male & Female \\
\hline G1 & Control & 0 & 1.0 & $5(1101-1105)$ & $5(2101-2105)$ \\
\hline G2 & TA & 1.0 & 1.0 & $5(1201-1205)$ & $5(2201-2205)$ \\
\hline
\end{tabular}




\subsubsection{Observation and inspection}

On the day of injection, day 0 , the general conditions, including toxicity indication type, toxicity expression and recovery times, and any deaths, were observed at $30 \mathrm{~min}-$ utes, and 1, 2, 4, and 6 hours after injection. Their general conditions were observed once daily, from day 1 to day 14 after injection. The animals' body weights were measured before injection on day 0 , and on days 3,7 , and 14 after injection.

\subsubsection{Histopathology}

After the observation period, all animals were anesthetized with $\mathrm{CO} 2$ and exsanguinated through the abdominal aorta. Complete postmortem examinations were performed for all animals. During the autopsy, the injection sites were harvested and preserved in $10 \%$ neutral buffered formalin. For the histopathological evaluation, preserved tissues were trimmed, dehydrated, paraffin-embedded, and then sectioned and stained with hematoxylin and eosin. All residual tissues were preserved in $10 \%$ neutral buffered formalin.

\subsection{Statistical analysis}

Statistical evaluation of the single-dose intramuscular toxicity test was performed by analyzing the body weights. After testing for equipotentiality using the Folded-F test and finding equal variance, Student's t-test was performed ( $\mathrm{P}$ $<0.05, \mathrm{P}<0.01$ ). SAS version 9.3 (SAS Institute Inc., Cary, NC, USA) was used for all analyses.

\section{Results}

\subsection{Effects of TA extract on general health and body weight}
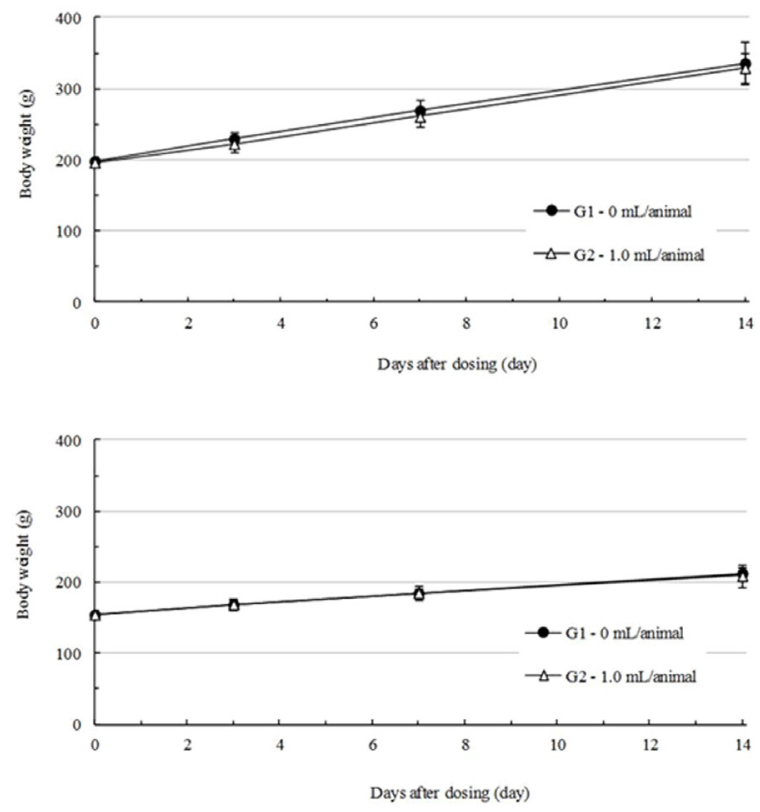

Figure 1 Effects of the TA extract on the body weight of male (A) and female (B) rats
During the observation period, there were no abnormalities, general symptoms of ill-health, or deaths in the male or female rats in the control and the TA-treated $(1.0 \mathrm{~mL})$ groups.

During the experiment, the weights of the male rats in the TA extract and the control groups changed from $198.4 \pm$ $5.8 \mathrm{~g}(\mathrm{G} 1)$ and $196.5 \pm 5.2 \mathrm{~g}(\mathrm{G} 2)$ to $336.2 \pm 29.0 \mathrm{~g}(\mathrm{G} 1)$ and $329.2 \pm 21.4 \mathrm{~g}(\mathrm{G} 2)$, respectively, while those of the female rats changed from $154.6 \pm 5.9 \mathrm{~g}(\mathrm{G} 1)$ and $154.5 \pm 5.9 \mathrm{~g}$ (G2) to $212.8 \pm 8.9 \mathrm{~g}(\mathrm{G} 1)$ and $209.0 \pm 16.6 \mathrm{~g}$ (G2), respectively. However, there were no statistically significant changes in body weight associated with TA injection (Figure 1, Table 3).

Table 3 Effects of TA extract on the mean body weights in the single-dose intramuscular toxicity test in rats

\begin{tabular}{|c|c|c|c|c|c|c|c|}
\hline \multirow{3}{*}{ Sex } & \multirow{2}{*}{\multicolumn{2}{|c|}{$\begin{array}{c}\text { Group/ } \\
\text { Dose (mL/animal) }\end{array}$}} & \multicolumn{4}{|c|}{ Days after dosing } & \multirow{3}{*}{$\begin{array}{c}\text { Gain }(\mathrm{g}) \\
0-14 \\
137.8\end{array}$} \\
\hline & & & \multirow{2}{*}{$\begin{array}{c}0 \\
198.4\end{array}$} & \multirow{2}{*}{$\begin{array}{c}3 \\
229.8\end{array}$} & \multirow{2}{*}{$\begin{array}{c}7 \\
269.5\end{array}$} & \multirow{2}{*}{$\begin{array}{c}14 \\
336.2\end{array}$} & \\
\hline & G1 & Mean & & & & & \\
\hline & 0 & S.D. & 5.8 & 8.4 & 15.7 & 29.0 & 26.9 \\
\hline \multirow[t]{6}{*}{ Male } & & $\mathrm{N}(\mathrm{g})$ & 5 & 5 & 5 & 5 & 5 \\
\hline & $\mathrm{G} 2$ & Mean & 196.5 & 222.6 & 260.7 & 329.2 & 132.7 \\
\hline & 1.0 & S.D. & 5.2 & 11.5 & 13.3 & 21.4 & 16.8 \\
\hline & & $\mathrm{N}(\mathrm{g})$ & 5 & 5 & 5 & 5 & 5 \\
\hline & G1 & Mean & 154.6 & 168.7 & 184.4 & 212.8 & 58.2 \\
\hline & 0 & S.D. & 5.9 & 6.6 & 6.9 & 8.9 & 5.3 \\
\hline \multirow[t]{4}{*}{ Female } & & $\mathrm{N}(\mathrm{g})$ & 5 & 5 & 5 & 5 & 5 \\
\hline & $\mathrm{G} 2$ & Mean & 154.5 & 169.0 & 185.0 & 209.0 & 54.5 \\
\hline & 0 & S.D. & 5.9 & 7.2 & 10.2 & 16.6 & 15.7 \\
\hline & & $N(g)$ & 5 & 5 & 5 & 5 & 5 \\
\hline
\end{tabular}

\subsection{Effects of the TA extract on visual autopsy findings}

During autopsy, no remarkable findings were noted in the male and female rats, in the control or the TA-treated groups.

\subsection{Effects of the TA extract on histology of the injection sites}

Local tolerance of TA was tested by microscopic examination of the prepared tissue obtained from the injection sites of all animals. Microscopic examination did not reveal any TA-related changes in this study.

\section{Discussion}

Evaluation of non-clinical toxicity of medicinal plants and herbal medications, forms the basis for demonstrating the safety of these preparations and is also essential for the approval of clinical trials. Currently, a variety of pharmacopuncture medications are used in traditional clinics and several pharmacopuncture preparations have been developed, based on various literature reports and clinical expe- 
rience. Pharmacopuncture medicines require non-clinical and clinical assessments of efficacy and safety to achieve scientific recognition.

The Korea Food and Drug Administration requires nonclinical data on safety, including genotoxicity, single-dose toxicity, and repeated-dose toxicity, as well as non-clinical data on efficacy, to approve clinical trials of herbal Korean medicines [9-12]. Scutellariae Radix and Phellodendri Cortex are listed in the Korean Pharmacopoeia and Aucklandiae Radix, Aquilariae Lignum, and Carthami Fructus are listed in the Korean herbal medicine, standard edition, endorsed by the Korea Food and Drug Administration. Therefore, safety data for each herbal constituent may not be required for the evaluation of TA. However, the route of administration is not oral, but rather through am acupuncture point. Therefore, toxicity evaluation should be performed, to establish the scientific basis for its action and assess the safety of this fairly new medication. Although the safety of its constituents has been verified by the Korea Food and Drug Administration, it is essential to carry out a toxicological evaluation study, in order to establish scientific and public trust in the safety of TA $[1,2]$.

The single-dose toxicity test, qualitatively and quantitatively, evaluates toxicity that occurs within a short period of time, when a test substance is administered to a test animal in a single dose. Although animal species with metabolic efficiency similar to humans are preferred while conducting toxicity assessments, they may be difficult to perform, at the single-dose toxicity test stage. Therefore, rats are most commonly preferred among rodents and dogs are most commonly preferred among non-rodents. Moreover, sex-related differences in the manifestation of toxicity, may exist, depending on the drug. Therefore, one or more animals of both sexes, must be evaluated for toxicity $[12,13]$. Therefore, we evaluate the safety of $1.0 \mathrm{~mL} \mathrm{TA}$ injection in 10 male and 10 female SD rats in this study, with 10 rats in the TA-treatment and control groups. For 14 days after the administration of the TA extract, general conditions were observed and body weight was measured. Subsequently, euthanasia was performed for autopsy and local examination of the administration site. We found no changes in general conditions, weight, or histology caused by single-dose intramuscular injection of the TA extract in either, male or female rats. There were also no changes in tissues or organs due to the injection, and no deaths or abnormalities were observed in any of the rats. Moreover, no significant differences were observed in body weight during the 14-day observation period after injection of the TA extract. Thus, pharmacopuncture with TA extract at $1.0 \mathrm{~mL}$ appears to be safe in animals and humans.

Although this study indicates that pharmacopuncture with TA extract is safe for administration at a dose of 1.0 $\mathrm{mL}$ or less, studies on the adverse- effects have not been officially reported yet. Moreover, since this study only investigated the effect of a single-dose injection over a 14day observation period, further studies on its long-term effects and the effects of multiple doses are required before pharmacopuncture with TA extract can be established as an effective analgesic for patients with pain. If additional toxicity studies, such as in vitro chromosome aberration tests and in vitro mouse lymphoma TK assays, are required to firmly establish the safety of using TA extract for phar- macopuncture in clinical practice.

\section{Conclusions}

In conclusion, our study demonstrated that the safe, non-toxic dose of TA extract may be considered to be 1.0 $\mathrm{mL}$ or less. Thus, the TA extract may be safe for pharmacopuncture in patients in traditional medicine clinics.

\section{Conflicts of interest}

The authors declare that they have no conflicts of interest.

\section{Authors' contributions}

C Jung designed all experiments. C Jung and JH Hwang performed the statistical analysis, interpreted the experimental results. JH Hwang wrote the manuscript. JH Hwang and HW Jung revised the manuscript. All authors read and approved the final manuscript.

\section{Acknowledgment}

This work was supported by from the National Research Foundation of Korea (NRF) grant funded by the Korean government (MSIT) (No. NRF- 2017R1C1B5076224).

\section{Availability of Data and Material}

The data set generated and analyzed during the study is available from the corresponding author on request. All materials used in this study are accurately recorded in the Methods section. 


\section{References}

1. Korean Acupuncture \& Moxibustion Society: Pharmacopuncture therapy. In: Acupuncture Medicine. Kim JH (ed.) Hanmi Medical Publishing Co., Paju. 2016.

2. Jung C, Jung JH, Lee MS. Pharmacopuncture medicines. In: A Clinical Study of Immune Pharmacopuncturology. Jung C (ed.) Chungnam: Kyungrak Medical Publishing Co. 2011:127-33.

3. Im WH, Jeong SH, Lim YH, Jung C, Kim HJ. Anti-inflammatory activities of $\mathrm{V}, \mathrm{TA}$ and $\mathrm{SH}$ Yakchim on LPS-induced nitric oxide and pro-inflammatory cytokines production in Raw 264.7 macrophages. J Immuno-Pharmacopuncture. 2016;5(1):19-25.

4. Wijnstekers $W$. The Convention on International Trade in Endangered Species of Wild Fauna and Flora (CITES) - 35 years of global efforts to ensure that international trade in wild animals and plants is legal and sustainable. Forensic Sci Rev. 2011;23(1):1-8.

5. Chung YH, Lee YK, Lee HJ, Kim JS. A case report of thoracic pain which was occurred by seat belt with additional TA pharmacopuncture. J Immuno-Pharmacopuncture 2017;6:39-48.

6. Hwang JH, Jung HW. TA pharmacopuncture as a primary and independent treatment for frequent sprains occurring over 9 months in a patient with needle sickness: Case report. Medicine. 2018;97:45.

7. Jung C, Hwang JH. Safety study of TA pharmacopuncture: Bacterial reverse mutation test. J Immuno-Pharmacopuncture. 2017;6:1-11.

8. Jung C, Hwang JH. Safety study of TA pharmacopuncture: In vitro chromosome aberration test using mammalian cultured cells. J Immuno-Pharmacopuncture. 2017;6:13-20.

9. KFD: Good Laboratory Practice (GLP), 2017. http: / / www.law.go.kr/admRulInfoP.do? admRulSeq=2100000086689

10. KFDA: Partial amendment official announcement about toxicity test for drug. 2015.

11. Lee MY, Seo CS, Kim JY, Shin HK. Genotoxicity evaluation of Guibi-Tang extract using an in vitro bacterial reverse mutation assay, chromosome aberration assay, and in vivo micronucleus test. BMC Complement Altern Med. 2014;14:215.

12. Sung H, Lee E. Single-dose intramuscular toxicity of Mahwangcheonoh pharmacopuncture in a rat model: Toxicity of Mahwangcheonoh pharmacopuncture in SD rats. J Pharmacopuncture 2016;19(4):336-43.

13. Kirsch-Volders M, Sofuni T, Aardema M, et al. Report from the in vitro micronucleus assay working group. Mutat Res. 2003;540(2):153-63. 